\title{
Comprehensibility of Newly Introduced Water-sport Prohibitive Signs in Korea by Koreans and Westerners
}

\author{
Woojoo Kim, Yohana Siswandari, Shuping Xiong \\ UNIST, Department of Human and Systems Engineering Ulsan, 689-798
}

\author{
Corresponding Author \\ Shuping Xiong \\ UNIST, Department of Human and \\ Systems Engineering Ulsan, 689-798 \\ Mobile: $+82-10-4222-2625$ \\ Email : maverickhkust@unist.ac.kr
}

Received : December 03, 2014

Revised : December 31, 2014

Accepted: February 02, 2015
Objective: The goal of this study is to evaluate the comprehensibility of the newly introduced water-sport prohibitive signs by the Ministry of Knowledge Economy (MKE, later merged into the Ministry of Trade, Industry and Energy) among Koreans and westerners, and to check whether the comprehensibility is affected by cultural differences.

Background: The Ministry of Knowledge Economy had newly introduced fourteen water-sport prohibitive signs at the end of 2011 to alert people to potentially dangerous situations. However, no studies had been found so far to review or assess their comprehensibility.

Method: Comprehensibility tests of fourteen water-sport prohibitive signs were conducted with forty Koreans and forty Westerners in two sequential sessions. In session I, participants were asked to guess the meaning of each sign verbally in an open-ended test. In session II, participants were encouraged to provide feedback for each sign after its intended meaning was given.

Results: Only two out of fourteen signs satisfied the comprehension rate (67\%) recommended by ISO standard for both groups (Koreans and Westerners). Cultural difference between Koreans and westerners significantly affect the comprehension rates of the investigated signs, and Westerners exhibit better overall comprehension than Koreans. Five poorly comprehended signs for both Korean and Western groups were identified.

Conclusion: The recently introduced water-sport prohibitive warning signs by MKE still need a lot of improvements in order to be implemented nationally or internationally. There were significant differences in the signs' comprehensibility between Koreans and westerners.

Application: The findings may serve as a useful input for researchers and watersport sign designers in creating easy-to-comprehend safety signs.

Keywords: Comprehensibility, Prohibitive signs, Cultural difference, Safety, Water-sport

\section{Introduction}

of Korea. All right reserved.

(C) This is an open-access article distributed under the terms of the Creative Commons Attribution Non-Commercial License (http:// creativecommons.org/licenses/by-nc/3.0/), which permits unrestricted non-commercial use, distribution, and reproduction in any medium, provided the original work is properly cited.
Prohibitive symbols refer to symbols with negative messages, frequently conveying information about actions that should not be taken in specific situations, or about conditions that should be prevented (Shieh and Huang, 2003). Usually, these kinds of symbols are found in public places and one of typical examples of prohibitive symbols is traffic signs symbols. However, these symbols have also been widely used 
in other public places, for example, beaches. By the end of 2011, the Ministry of Knowledge Economy (MKE, later merged into The Ministry of Trade, Industry and Energy) of South Korea (http://www.mke.go.kr) had introduced a set of water-sport prohibitive signs to alert people to potentially dangerous situations. Considering the possible dangerous situations that might happen should the beachgoers failed to understand the meaning of these signs, it is necessary to investigate the comprehensibility of these signs by their prospective users.

Even though Korean citizens will be the major intended users and their comprehension of the recently introduced signs is of utmost importance, the comprehension of those signs by Westerners was also investigated in this study due to two main reasons. First, it has been reported that the number of migrant workers residing in Korea had doubled from the number reported in 2006 (more than a half million) and reached approximately 1.1 million in 2008, and 1.4 million in 2012 (Kim, 2009). Thus, multinational beachgoers are becoming more common in South Korea. Therefore, it will be good if the introduced water-sport prohibitive signs can convey their intended information to the beachgoers regardless of their nationalities, which is to say the signs should be culture-independent, or culturally compatible (Galdo, 1990). Second, previous studies have reported significant differences in perception and ways of thinking between western and Asian cultures (Cairney and Sless, 1982; Wu and Keysar, 2007; Savani et al., 2008; Falk et al., 2009). For example, East Asians have been found to differ in their choice making from Americans: slower to make choices, less likely to choose according to their preferences, and less motivated to express their preferences in their choices (Savani et al., 2008). Cairney and Sless (1982) also found that Australians and Vietnamese demonstrated different recognition and recall with regard to industrial-safety signs. Due to the importance of cross-cultural issues in sign design research (Dewar 1994; Ben-Bassat and Shinar, 2006; Ou and Yung, 2012), whether the cultural differences as pointed out by former studies affect the comprehensibility of the safety signs introduced by MKE is worthy of further investigation.

For safety signs which have been implemented for several years, there should be no serious problems since most people may have adequate experience of comprehending those signs (Ben-Bassat and Shinar, 2006). In case of the newly introduced prohibitive signs, comprehension problems are likely to arise due to the lack of adaptation effect. However, until this study was conducted, there has been no indication from MKE that these signs have been assessed and standardized nationally, nor internationally. Moreover, no studies had been found so far to review or assess the comprehensibility of these newly introduced water-sport prohibitive signs. In coherence to this, the current study was aimed to evaluate the comprehensibility of the newly introduced water-sport prohibitive signs established by MKE among Koreans and westerners, and to check whether the comprehensibility is affected by cultural differences.

\section{Method}

\subsection{Participants and stimuli}

In total, eighty healthy young adults (18-35 years old) consisting of forty Koreans and forty Westerners voluntarily participated in this experiment. A westerner was defined as a native or inhabitant of the western world (Millar et al., 2013). The forty westerners in this study hold the nationalities of these countries: United States of America(10), United Kingdom(9), Canada(8), The Netherlands(4), Australia(2), Germany(2), Portugal(2), France(1), Spain(1), and New Zealand(1). The inclusion criteria were that they should have normal or corrected-to-normal vision, have no color blindness, and have no previous experience of learning the actual meanings of the water-sport signs tested here. In addition, all western subjects who are not native English speakers should have a TOEFL score of at least 90 (internet-based), or original academic IELTS score of at least 6.5. This criterion was set to assure that all westerners have sufficient English language proficiency to participate in the experiment.

Out of fifteen newly introduced water-sport safety signs issued by the MKE, fourteen signs which serve as prohibitive signs were used as stimuli (Figure 1). Even though in reality, the messages of the sign symbols could be also conveyed with Korean text, 
only the pictorial symbols were shown in this experiment, because pictorial symbols can quickly communicate concepts and may be better remembered than text (Rogers et al., 2000; Lesch, 2003; Hancock et al., 2004). Pictorial symbols are also useful in conveying safety information to members of diverse educational levels (Kim et al., 2006) and cultures (Lesch, 2003; Hancock et al., 2004) since non-native Korean speakers need not rely on their knowledge of the Korean language to correctly interpret symbols. These signs were fitted into $7 \mathrm{~cm}{ }^{*} 7 \mathrm{~cm}$ squares without boundary, at a distance of $60 \mathrm{~cm}$ (subtending $6.67^{\circ}$ from the screen) in a randomized order ( $\mathrm{Ng}$ and $\mathrm{Chan}, 2007$; Liu and Ho, 2012). To comply with the angle requirement, an adjustable chair was used for the participants.

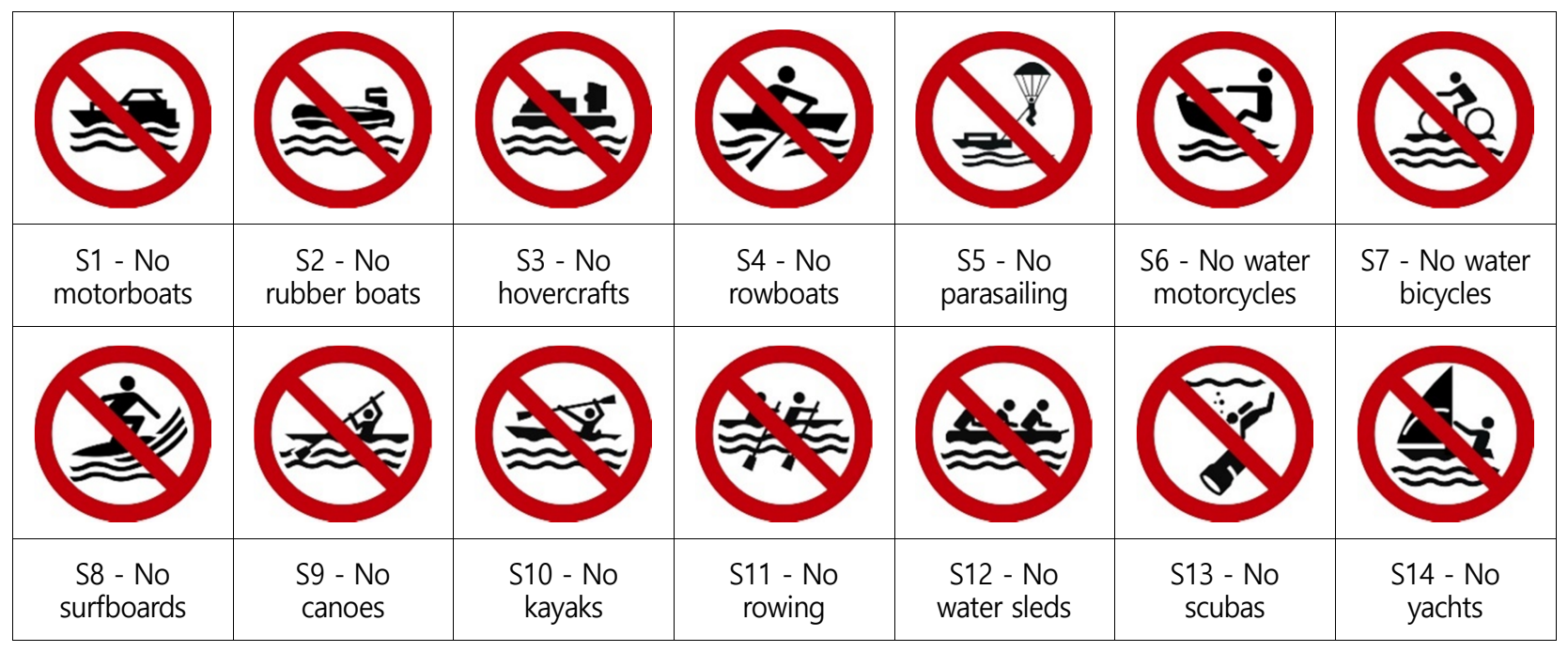

Figure 1. Recently introduced fourteen water-sport prohibitive signs by the Ministry of Knowledge Economy (MKE) of Korea (http://www.MOTIE.go.kr/common/)

\subsection{Experimental procedure}

Ishihara color blindness test was conducted to screen out participants with color-blindness. After each qualified participant filled out the consent form, an experiment consisting of two sequential sessions was conducted and it lasted for approximately 1.5 hours. To obtain reliable experimental data from both the Korean and western groups, the whole experiment was conducted in Korean for Koreans and in English for westerners.

In session I, participants were asked to guess the actual meaning of each sign in an open- ended test. The decision to let participants speak out the meaning of the signs in their own words (open-ended) rather than using multiple-choice options was made because this approach has been proven to yield greater ecological validity (Wolff and Wogalter, 1998; Ou and Yung, 2012). One sign was displayed at once on the computer screen. Each participant was given a chance to look at the signs for 10 seconds, and then give the answers verbally within 15 seconds. After they finished giving answers for one sign, the next sign was presented. This procedure was repeated for all fourteen signs in randomized order.

In session II, participants were encouraged to give feedback for each sign. Given the actual meaning associated with each displayed sign, each participant was asked to give a detailed opinion on each sign's features (size, shape, orientation, ambiguous object depicted in the sign, etc) and/or ideas for general improvement. A sketchbook and drawing tools were also provided in 
case they wanted to express their opinions by drawing. This procedure was repeated until all fourteen signs had been evaluated. The whole experiment sessions were recorded by a video camera and a monitor recording program (oCam version 13.0). At the end of the experiment, participants were asked to fill out a questionnaire to capture information related to their experiences with water sports.

\subsection{Data processing and statistical analysis}

Participants' comprehension of each tested sign was measured by guessability score (the accuracy level of guessing the meaning of a sign) in the range of 0 to 100\% (ISO 9186-1, 2007; $\mathrm{Ng}$ and Chan, 2007), which was determined from the recorded verbal responses by two judges. The correct understanding of the actual meaning of the sign symbol was given $100 \%$ and the scoring procedure was carried out independently by each judge. An Intraclass Correlation Coefficient (ICC) analysis was conducted to check whether the two independent judges had a high level of agreement on assessing the accuracy of participants' responses. The ICC $(2,2)$ coefficient was 0.970 , indicating a high level of consistency among the judges. Thus, the averaged data from two judges was used for further analysis.

Two-sample $t$-tests and correlation analysis were conducted to check the differences and similarities in the comprehension of safety signs between the two groups (Koreans and westerners). Repeated measures Analysis of Variance (ANOVA) was conducted for Korean and western groups separately, to see whether there was a significant difference in the guessability among different signs. A Bonferroni post hoc test was done for each group (Koreans and westerners) to categorize the tested signs based on their guessability scores. Two widely reported international standards (International Organization for Standardization-ISO, and American National Standard Institute-ANSI) for safety symbols were used for further determining which signs can be considered acceptable to be applied in South Korea (locally) and globally. According to ISO and ANSI, symbols must reach a criterion of at least $67 \%$ or $85 \%$ correct, respectively, in a comprehension test to be considered acceptable (Wolff and Wolgater, 1998). Feedback obtained from all participants was utilized to analyze causes for poorly comprehended signs.

\section{Results}

\subsection{Guessability scores among Korean and western participants}

The descriptive statistics of guessability scores for all 14 signs are shown in Figure 2. The overall mean (standard error) of guessability scores for all signs was $43.0 \%$ (3.5\%) and $58.3 \%$ (4.3\%) for Koreans and westerners, respectively. Two-sample t-tests show that there were significant $(p<0.05)$ cultural differences in the comprehensibility of 8 out of 14 signs (Figure 2), including S1-no motorboats, S2-no rubber boats, S4-no rowboats, S5-no parasailing, S6-no water motorcycles, S9-no canoes, S10-no kayaks, S14-no yachts. Surprisingly, in all cases, the signs were guessed accurately more often by western participants than by Korean participants. Even though there were significant differences between the two user groups in the comprehension of several signs, there were also similarities. A correlation analysis (Figure 3) showed that there were significant correlations between the average guessability scores of each sign given by Korean and western participants. The correlation between overall comprehension levels of Koreans and westerners was high $(r=0.866)$. This means that signs that are not understood very well by Korean participants may not be understood very well by western participants either, and the other way around.

The ANOVA results showed that the guessability score differed significantly among different signs for both the Korean group $[F(13,39)=17.44, p<0.0001]$ and the western group $[F(13,39)=26.37, p<0.0001]$. Further Bonferroni post hoc grouping analysis (Figure 4) showed that five signs (S1-No motorboats, S2-No rubber boats, S3-No hovercrafts, S7-No water bicycles, and S12-No water sleds) were classified as the most or the second most difficult to comprehend groups consistently across both Korean and western participants. Additionally, the average guessability scores for each of them were below $50 \%$ (getting close 


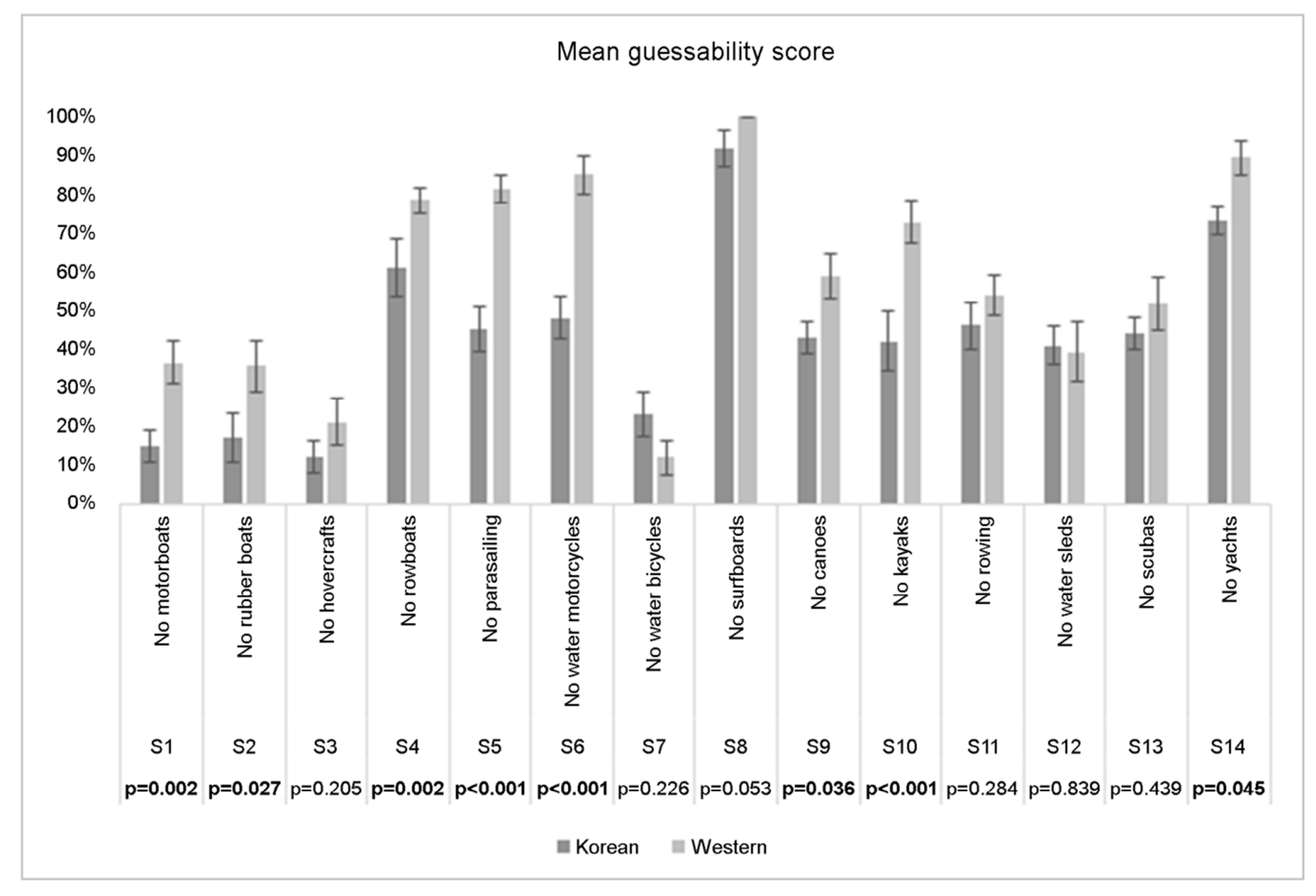

Figure 2. Descriptive statistics (mean and standard error) of guessability scores for 14 safety signs in Korean and western groups ( $p$-value for group difference is bolded if less than 0.05)

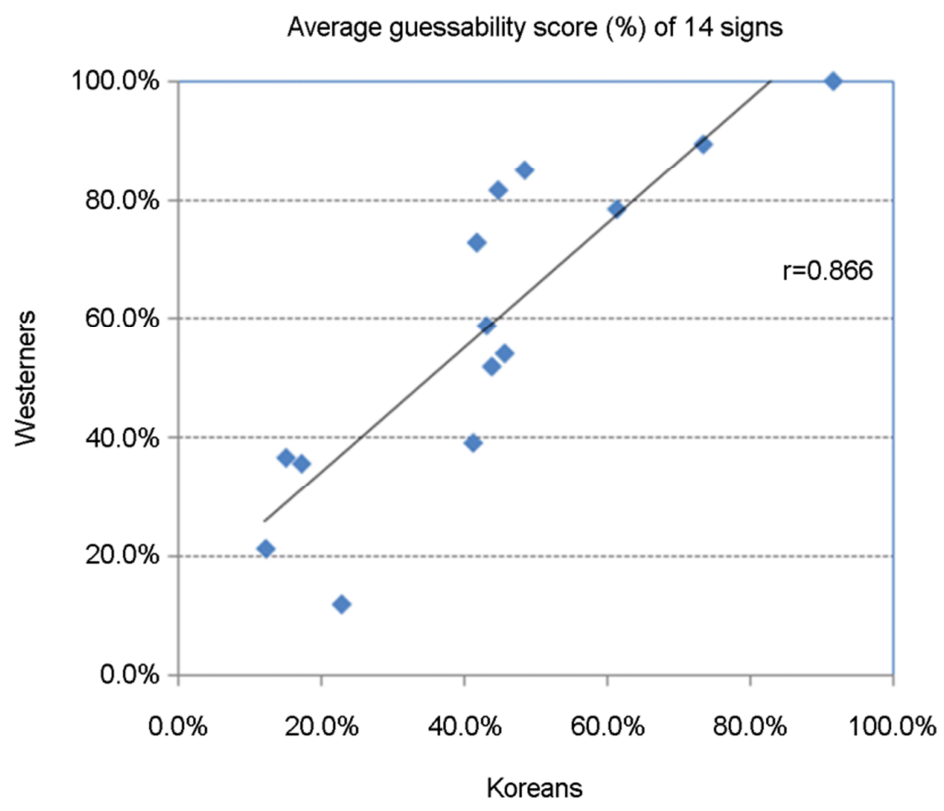

Figure 3. The relationship between Koreans' and westerners' comprehension (average guessability score) of 14 safety signs 
to the actual meaning) for both Korean and western groups, they should be considered as poorly comprehended safety signs.

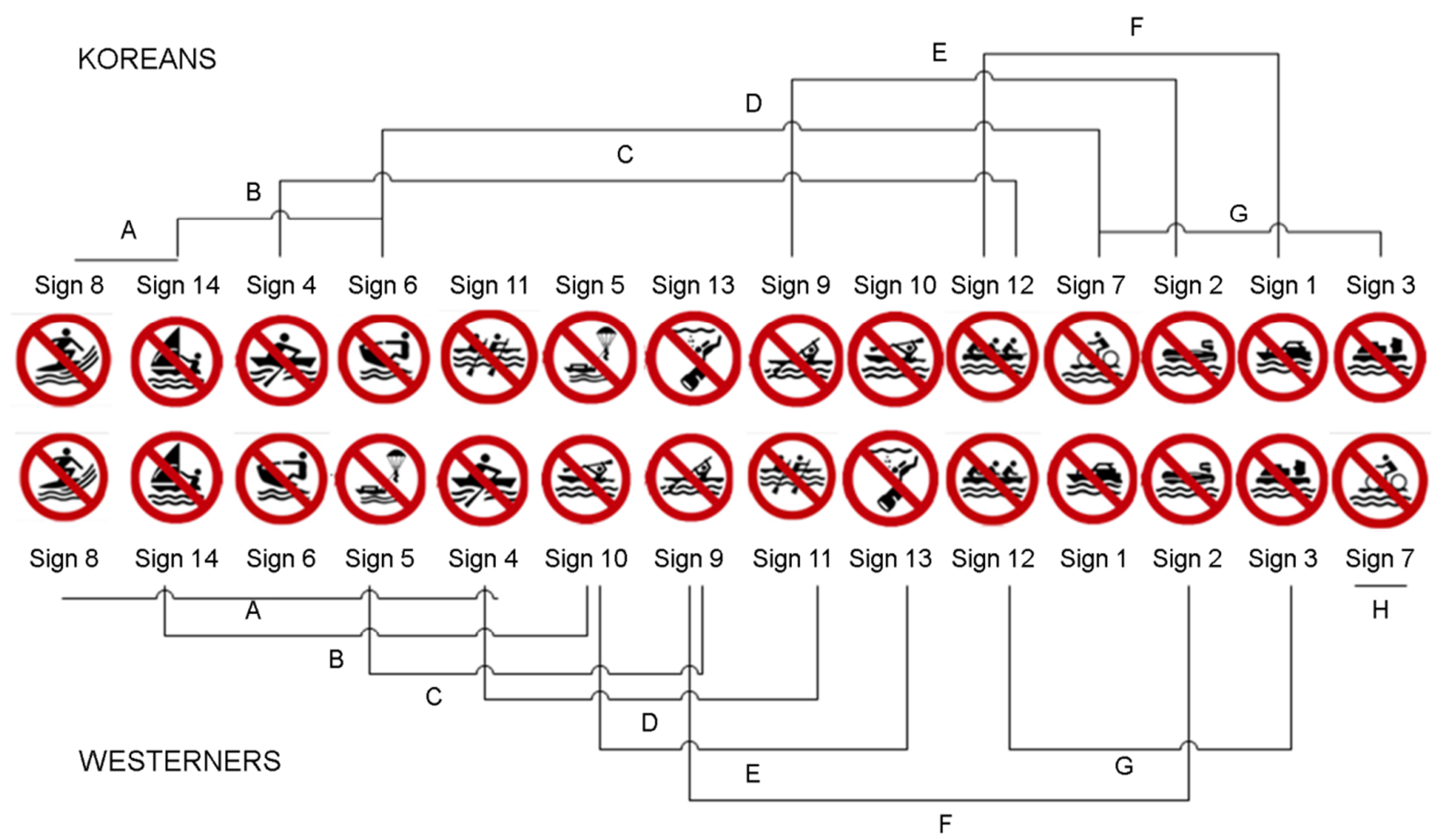

Figure 4. Grouping signs based on guessability scores (Letter difference indicates significance)

\subsection{Signs that conform to international standards for each group}

Considering both ISO and ANSI standards (guessability score must be at least 85\%), only sign 8 (no surfboards) can be applied for Koreans, while for westerners sign 8 (no surfboards) and sign 14 (no yachts) are considered acceptable. On the other hand, if we consider the ISO standard alone (guessability score must be at least 67\%), sign 8 and sign 14 are acceptable to be applied for Koreans; while for westerners, sign 8, sign 14, sign 6 (no water motorcycles), sign 5 (no parasailing), sign 4 (no rowboats), and sign 10 (no kayaks) are acceptable. Combining the results together, only sign 8 (no surfboards) and sign 14 (no yachts) conform to the ISO standard and can be applied both nationally and internationally.

\section{Discussion}

The main purpose of this study is to evaluate the comprehensibility of recently introduced water-sport prohibitive signs by MKE in South Korea among local people (Koreans) and westerners, and to assess the effect of cultural differences on the comprehensibility of these signs. The experimental results showed that there were significant cultural differences between Koreans and westerners in comprehension of the same water-sport safety signs. Previous cross cultural studies also reported significant cultural differences in sign comprehension (Al-Madani and Al-Jahani, 2002; Shinar et al., 2003; Lee et al., 2014). A study by Lee et al., which administered 19 comprehension tests for Americans, Koreans, and Turkish, demonstrated that people comprehended symbols from their own country significantly better (Lee et al., 2014). Hence, it was hypothesized that Koreans could better understand the tested signs, compared to the westerners. However, contrary to our expectation, this study found that westerners 
had better understanding of the recently introduced water-sport safety signs issued by MKE than the Koreans, who are supposed to be the major intended users. These surprising results could be due to two main reasons as follows.

Firstly, some signs were designed exactly the same as, or at least similar to, water-sport safety signs that have already been issued in western countries, specifically UK and Australia. As shown in Figure 5a, the signs "no motorized craft", "no personal watercraft", "no surfboarding", and "no rowing" were adopted for sign 1 (no motorboats), sign 6 (no water motorcycles), sign 8 (no surfboards), and sign 4 (no rowboats). Meanwhile, the sign "no sub-aqua equipment" was similar to sign 13 (no scubas). Aside from the United Kingdom water safety signs, some signs from the Australian national aquatic and recreational signage style manual (2006) showed a similar style with the recently introduced signs. For example, the sign "rafting" (Figure 5b) is similar to sign 12 (no water sleds), with minor differences such as angle and a small part in front of the boat. Actually, this might also be the reason why some western participants misunderstood the sign "no water sleds" to mean "no rafting". Thus, some recently introduced signs by MKE might be directly adapted from those countries, which are less likely to consider culture and perception of the Korean population, even though significant cultural differences in sign comprehension were reported in previous studies (Al-Madani and Al-Jahani, 2002; Shinar et al., 2003).

a)

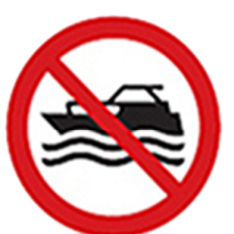

No motorized craft

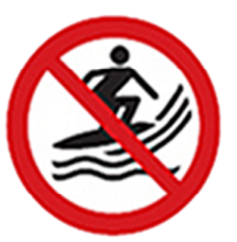

No surfboarding

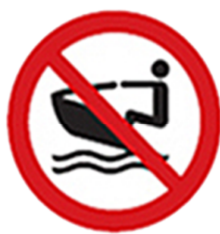

No personal watercraft

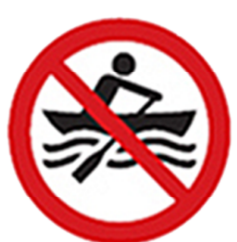

No rowing

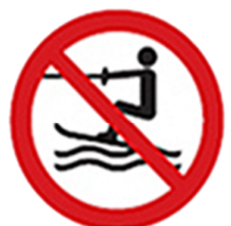

No water-skiing

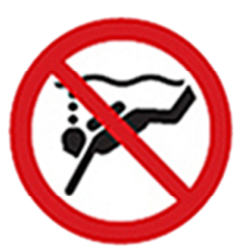

No sub-aqua equipment b)

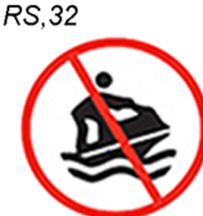

PWC's

PROHIBITED

$R S, 37$

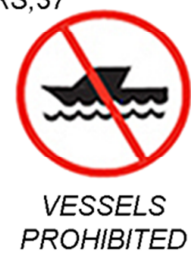

RS, 35

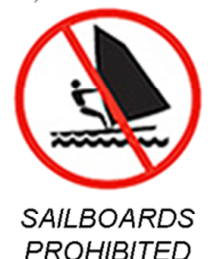

RS, 17

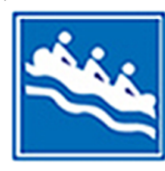

RAFTING

Figure 5. United Kingdom water safety signs used in workplaces and public areas, published September 2011 (a), Australian national aquatic and recreational signage style manual, published July 2006 (b)

Secondly, participants' prior experience with water sports is likely to be one reason why the westerners performed better in the guessing test, especially considering water sports might have been more incorporated into the western culture and lifestyle. Statistical analysis on questionnaire data of water-sport related experience was conducted to prove this hypothesis. The results showed that significant $(p<0.001)$ difference on the number of water sports played between the Korean and western groups. On average, Korean participants experienced $1.68( \pm 1.42)$ types of water sports, while western participants experienced $6.40( \pm 3.54)$ types of water sports. In terms of frequency of visiting the beach, again, western participants tend to visit the beach more frequently than Korean participants, indicating that the westerners had more prior experience than the Koreans. Questionnaire data showed that 19 out of 40 western participants go to the beach more than three times a year, and 11 people go to the beach more than 10 times a year. On the other hand, 20 out of 40 Korean participants go to the beach less than three times a year, and only one participant frequently visits the beach (more than 10 times a year). Previous studies also reported that personal variables including prior experience/familiarity with safety signs can significantly affect the comprehension of the signs (Rogers, 
Lamson and Rousseau, 2000; Ben-Bassat and Shinar, 2006). This study found that prior experience (familiarity) with water-sports helped the westerners understand the meanings of the signs better. This finding is consistent with most previous studies which have reported a high and significant positive correlation between sign comprehension and years of related experience, especially in the field of transportation (Ben-Bassat and Shinar, 2006). This study also suggests that comprehension probability is somehow associated with a learning process. Simply speaking, when a sign is more familiar or encountered more frequently, users have a better opportunity to learn its meaning and retain that information in memory, thus making the sign itself easier to comprehend (Rogers, Lamson and Rousseau, 2000).

Only two and six out of the fourteen safety signs have been found acceptable to be applied for Koreans and westerners, respectively, based on the ISO standard (at least 67\% comprehension level), indicating that the recently introduced safety symbols need to be significantly improved, important text messages should be supplied with the symbols, or sufficient symbol training should be provided before implementation (Lesch, 2003; Hancock et al., 2004). While compliance with international standards is one important aspect in designing safety signs intended for multi-national users, creating an international standard in sign compatibility is challenging since cultural differences among the sign users could possibly influence how they associate the picture depicted on the sign with an object they are familiar with. In this study, some signs were poorly understood by both groups, Koreans and westerners, for different reasons. For example, sign 7 (no water bicycles) was not really comprehensible among westerners (guessability score $=11.9 \%$ ) given the fact that they were not familiar with water bicycles (22 out of 40 participants guessed this sign as "no cycling near the water" instead of "no water bicycles"). In the case of Korean participants, although they were able to define what 'water bicycle' means, they also got a low guessability score (guessability score $=22.8 \%$ ) mainly because of misunderstanding caused by the lower part of the picture. Instead of recognizing the lower part of the 'water bicycle' as a buoy, they recognized it as a path. Another example is sign 3 (no hovercrafts). This sign got low scores among both groups (westerners and Koreans). However, most westerners knew what a hovercraft looks like, while most Koreans did not. The low guessability score for the "no hovercrafts" sign was mainly caused by the sign's lack of resemblance to a real hovercraft for westerners (guessability score $=21.2 \%$ ), while for the Koreans, the low guessability score (guessability score $=12.2 \%$ ) for this sign was the result of their limited understanding of what a hovercraft looks like. Another interesting finding was discovered for sign 14 (no yachts). For this sign, there is a terminology difference between the Koreans and westerners. The Korean use of the term "yacht" includes all sailing boats, but for westerners "yachts" and "sailing boats" are two completely different things. This occurrence could cause a difference in semantic closeness between the Korean and western groups for this particular sign. Nevertheless, westerners showed a high average guessability score (89.4\%), even higher than the Koreans' average guessability score $(73.4 \%)$, indicating that the sign can actually convey its intended meaning very well regardless of the terminologies used in different cultures.

There are several limitations inherent in this study. First, the signs used in this research were presented to subjects in the absence of context, and the subjects probably would have performed better in the guessing test if the signs were presented in appropriate contexts. Second, participants' prior experience related to water-sport activities and other characteristics cannot be fully controlled in this study, inducing possible confounding variables to cultural differences on the sign comprehension. Third, the identified poorly comprehended safety signs should be redesigned for better comprehension. We are revising those signs using ergonomic design principles and obtained feedback from all participants, the revised signs will be further assessed and verified for their effectiveness in the near future.

\section{Conclusion}

The present study investigated the comprehensibility of fourteen recently introduced water-sport prohibitive signs by MKE with forty Koreans and forty Westerners. Results showed that the introduced prohibitive warning signs still need a lot of improvements in order to be implemented nationally or internationally. Significant differences in the signs' comprehensibility between Koreans 
and westerners were also found, and contrary to our expectation, the westerners exhibited better overall comprehension for the sign set than Koreans, who are the major intended users. Participants' prior experience in related fields was associated with the enhanced comprehensibility of the safety signs. The findings of this study may serve as a useful input for water-sport sign designers in creating easy-to-comprehend safety signs.

\section{Acknowledgements}

This study was funded by the Basic Science Research Program through the National Research Foundation of Korea (NRF 2011 0022185; NRF 2014-056193). The authors would like to give special thanks to Jung-Min Park for his help on data acquisition in this experiment.

\section{References}

Al-Madani, H. and Al-Jahani, A.R., Assessment of drivers' comprehension of traffic signs based on their traffic, personal and social characteristics. Transportation Research Part F: Traffic Psychology and Behaviour, 5(1), 63-76, 2002. doi: 10.1016/S13698478(02)00006-2

Ben-Bassat, T. and Shinar, D., Ergonomics guidelines for traffic sign design increase sign comprehension. Human Factors, 48(1), 182-195, 2006. doi: 10.1518/001872006776412298

Cairney, P. and Sless, D., Communication effectiveness of symbolic safety signs with different user groups. Applied Ergonomics, 13, 91-96, 1982. doi: 10.1016/0003-6870(82)90185-5

Dewar, R.E., Design and evaluation of graphic symbols. In Proceedings of Public Graphics. Utrecht, Netherlands: University of Utrecht, Department of Psychonomics, 1994.

Falk, C.F., Heine, S.J., Yuki, M. and Takemura, K., Why do Westerners self-enhance more than East Asians? European Journal of Personality, 23, 183-209, 2009. doi: 10.1002/per.715

Galdo, E.D., Internationalization and translation: some guidelines for the design of human-computer interfaces. In: Nielsen, J. (Ed.), Designing User Interfaces for International Use (pp. 1-10). New York: Elsevier, 1990.

Hancock, H.E., Rogers, W.A., Schroeder, D. and Fisk, A.D., Safety symbol comprehension: Effects of symbol type, familiarity, and age. Human Factors, 46(2), 183-195, 2004. doi: 10.1518/hfes.46.2.183.37344

ISO 9186-1., Graphical symbols-test methods- part 1: methods for testing comprehensibility. Geneva: International Standards Organization (ISO), 2007.

Kim, A.E., Global migration and South Korea: foreign workers, foreign brides and making of a multicultural society. Ethnic and Racial Studies, 32(1), 70-92, 2009. doi: 10.1080/01419870802044197

Kim, D.H., Lee, J.W., Park, Y.W. and Lim, H.K., A questionnaire survey on utilization and improvement guides for safety signs in industrial fields. Journal of the KOSOS, 21(4), 119-126, 2006.

Lee, S., Dazkir, S.S., Paik, H.S. and Coskun, A., Comprehensibility of universal healthcare symbols for wayfinding in healthcare 
facilities. Applied Ergonomics, 45, 878-885, 2014. doi: 10.1016/j.apergo.2013.11.003

Lesch, M.F., Comprehension and memory for warning symbols: Age-related differences and impact of training. Journal of Safety Research, 34, 495-505, 2003. doi: 10.1016/j.jsr.2003.05.003

Liu, Y.C. and Ho, C.H., The effects of age on symbol comprehension in central rail hubs in Taiwan. Applied Ergonomics, 43, 1016-1025, 2012. doi: 10.1016/j.apergo.2012.02.004

Millar, P.R., Serbun, S.J., Vadalia, A. and Gutchess, A.H., Cross-cultural differences in memory specificity. Culture and Brain, 1(2-4), 138-157, 2013. doi: 10.1007/s40167-013-0011-3

$\mathrm{Ng}$, A.W. and Chan, A.H., The guessability of traffic signs: effects of prospective-user factors and sign design features. Accident Analysis \& Prevention, 39(6), 1245-1257, 2007. doi:10.1016/j.aap.2007.03.018

Ou, Y.K. and Yung, C.L., Effects of sign design features and training on comprehension of traffic signs in Taiwanese and Vietnamese user groups. International Journal of Industrial Ergonomics, 42, 1-7, 2012. doi: 10.1016/j.ergon.2011.08.009

Rogers, W.A., Lamson, N. and Rousseau, G.K., Warning research: An integrative perspective. Human Factors, 42(1), 102-139, 2000. doi: 10.1518/001872000779656624

Savani, K., Markus, H.R. and Conner, A.L., Let your preference be your guide? Preferences and choices are more tightly linked for North Americans than for Indians. Journal of Personality and Social Psychology, 95, 861-876, 2008.

Shieh, K. and Huang, S., Factors affecting preference ratings of prohibitive symbols. Applied Ergonomics, 34, 581-587, 2003. doi: 10.1016/S0003-6870(03)00078-4

Shinar, D., Dewar, R., Summala, H. and Zakowska, L., Traffic sign symbol comprehension: a cross-cultural study. Ergonomics, 46 (15), 1549-1565, 2003. doi: 10.1080/0014013032000121615

Sims, B., Water safety signs and beach safety flags. The handbook of drowning, 204-214, 2006.

Wolff, J.S. and Wogalter, M.S., Comprehension of pictorial symbol: effects of context and test method. Human Factors, 40, 173186, 1998. doi: 10.1518/001872098779480433

Wu, S. and Keysar, B., Cultural effects on perspective taking. Psychological Science, 18, 600-606, 2007.

\section{Author listings}

Woojoo Kim: xml1324@unist.ac.kr

Highest degree: Bachelor degree in Human and Systems Engineering

Position title: Master student, UNIST

Areas of interest: Safety sign evaluation and redesign; Physical ergonomics 
Yohana Siswandari: yohanapra@yahoo.com

Highest degree: Master degree in Human and Systems Engineering

Position title: None

Areas of interest: Comprehension of safety sign; Cognitive ergonomics

Shuping Xiong: maverickhkust@unist.ac.kr

Highest degree: PhD degree in Industrial Engineering

Position title: Associate Professor, UNIST

Areas of interest: Ergonomic design; Digital human modeling; Human balance and fall risk assessment; Applied biomechanics 